\title{
DINÂMICA DE CÁLCIO E MAGNÉSIO EM FOLHAS E FRUTOS DE Coffea arabica $^{(1)}$
}

\author{
Bruno Galvêas Laviola ${ }^{(2)}$, Hermínia Emilia Prieto Martinez ${ }^{(3)}$, \\ Ronessa Bartolomeu de Souza ${ }^{(4)}$ \& Víctor Hugo Alvarez V. ${ }^{(5)}$
}

\begin{abstract}
RESUMO
Conhecer a dinâmica de nutrientes minerais em cafeeiro permite identificar o período de maior exigência nutricional da planta e, assim, melhorar a eficiência das práticas de adubação. O objetivo deste trabalho foi estudar a dinâmica de Ca e Mg em frutos de cafeeiro da antese à maturação e compará-la à dinâmica desses elementos em folhas dos ramos produtivos. O experimento foi realizado com três variedades de Coffea arabica (Catuaí Vermelho IAC-99, Rubi MG-1192 e Acaiá IAC-474-19) distribuídas em três ensaios independentes (níveis de adubação baixo, adequado e alto), instalados em blocos ao acaso com duas repetições, em um esquema de parcelas subdivididas no tempo. As variedades apresentaram as maiores concentrações de Ca e Mg nos frutos no estádio de chumbinho, com redução na concentração desses elementos no estádio de expansão rápida. Nos estádios de crescimento suspenso e granação-maturação observou-se pouca ou nenhuma variação nas concentrações de Ca e Mg nos frutos. No $3^{\circ}$ e $4^{\circ}$ pares de folhas de ramos produtivos foram constatados decréscimos nas concentrações de $\mathrm{Ca}$ e $\mathrm{Mg}$ no início do período reprodutivo, havendo recuperação posteriormente. De modo geral, os níveis de adubação influenciaram a concentração de Ca e Mg em frutos e folhas das variedades de Coffea arabica ao longo do período reprodutivo. Contudo, as concentrações de Ca e Mg em folhas e frutos não foram influenciadas somente pelos níveis de adubação empregados, mas também por outros fatores que determinam a taxa de distribuição dos elementos minerais nas plantas de cafeeiros, como a carga pendente de frutos.
\end{abstract}

Termos para indexação: fisiologia vegetal, nutrição mineral.

\footnotetext{
(1) Parte da Tese de Mestrado do primeiro autor apresentada à Universidade Federal de Viçosa - UFV. Trabalho financiado pelo CNP\&D-Café. Recebido para publicação em dezembro de 2005 e aprovado em janeiro de 2007.

${ }^{(2)}$ Doutorando do Departamento de Fitotecnia, Universidade Federal de Viçosa - UFV. CEP 36570-000 Viçosa (MG). Bolsista CNPq. E-mail: laviolabg@yahoo.com.br

(3) Professora do Departamento de Fitotecnia, UFV. E-mail: herminia@ufv.br

(4) Pesquisadora da Embrapa Hortaliças. CNPH, CEP 70359-970 Brasília (DF). E-mail: ronessa@cnph.embrapa.br

(5) Professor do Departamento de Solos, UFV. E-mail: vhav@ufv.br
} 


\title{
SUMMARY: DYNAMICS OF CALCIUMAND MAGNESIUM IN LEAVES AND FRUITS OF ARABIC COFFEE
}

\begin{abstract}
Knowledge on mineral nutrient dynamics in coffee trees is important to identify the period of greatest nutritional requirement by the plant, allowing the improvement of fertilization techniques. The aim of our research was to study $\mathrm{Ca}$ and $\mathrm{Mg}$ dynamics in coffee fruits from anthesis to maturation and compare it with the dynamics of the same elements in leaves of productive branch leaves. The experiment was carried out with three Arabic coffee varieties (Catuaí Vermelho IAC-99, Rubi MG-1192 and Acaiá IAC-474-19) in three independent trials (low, sufficient and high fertilizer levels), arranged in randomized blocks with two replications using a split-plot scheme in time. The varieties presented the highest $\mathrm{Ca}$ and $\mathrm{Mg}$ fruit concentrations in the initial growth stage and decreasing concentrations in the stages of fast fruit expansion. In the stages of ceasing fruit growth and fruit formation-maturation little or no variation was observed in the Ca and $M g$ fruit concentrations. In the $3^{\text {rd }}$ and $4^{\text {th }}$ pair of leaves of the productive branches $\mathrm{Ca}$ and $\mathrm{Mg}$ concentrations decreased in the beginning of the reproductive period and recovered afterwards. In general, the fertilization levels influenced the Ca and $\mathrm{Mg}$ concentration in fruits and leaves of the varieties of Arabic coffee plant throughout the reproductive period. However, Ca and $\mathrm{Mg}$ concentrations in leaves and fruits were not only influenced by the fertilizer levels, but by other factors as well that determine the distribution rate of the mineral elements in the coffee plants, such as fruit load.
\end{abstract}

Index terms: plant physiology, mineral nutrition.

\section{INTRODUÇÃO}

O Brasil, é atualmente, o principal produtor mundial de café, tendo produzido na safra de 2006/07, de acordo com a CONAB (2006), 41,57 milhões de sacas de café beneficiado, com o Estado de Minas Gerais contribuindo com 50,9 \% da produção total. Contudo, a produtividade média brasileira ainda é baixa, tendo sido de apenas 20,96 sacas ha-1 de café beneficiado, considerando que o potencial produtivo das variedades está acima de 50 sacas ha-1 (Fazuoli et al., 2002). Dentre os principais fatores que contribuem para a produção do cafeeiro destaca-se a nutrição mineral, sendo importante o suprimento dos nutrientes e sua intensidade de absorção pelas raízes.

O Coffea arabica leva dois anos para completar o ciclo fenológico. De acordo com Gouveia (1984), no primeiro ano formam-se os ramos vegetativos com gemas axilares nos nós, durante os meses de dias longos. A partir de janeiro, quando os dias começam a encurtar, as gemas vegetativas axilares são induzidas por fotoperiodismo em gemas reprodutivas. O segundo ano fenológico do cafeeiro, de acordo com Camargo \& Camargo (2001), inicia-se com a floração, após choque hídrico nas gemas florais. Após a fecundação da flor, inicia-se o período de desenvolvimento do fruto, entre os meses de setembro e junho, passando pelos estádios de chumbinho, expansão rápida, granação até a maturação.

Durante a formação do fruto do cafeeiro e nos diversos estádios de desenvolvimento, há variações na concentração e no conteúdo dos elementos acumulados, assim como na produção de matéria seca. De acordo com Moraes \& Catani (1964), o consumo de nutrientes e o acúmulo de matéria seca são intensificados a partir do quarto mês após a floração. Segundo Matiello et al. (2005), a quantidade de nutrientes exigida nas fases de florada e chumbinho é pequena, aumentando, significativamente, a partir da passagem dos frutos para o estádio verde aquoso a verde sólido, na granação, até a maturação dos frutos. Cerca de $73 \%$ do crescimento vegetativo ocorre de outubro a abril, sendo o consumo de nutrientes para frutificação também concentrado nesse período (mais de $80 \%)$.

Chaves (1982) verificou que as concentrações de $\mathrm{N}, \mathrm{P}, \mathrm{K}, \mathrm{Ca}, \mathrm{Mg}, \mathrm{B}, \mathrm{Cu}, \mathrm{Mn}$ e $\mathrm{Zn}$ foram mais elevadas nos frutos nos estádios iniciais de crescimento, enquanto para o $\mathrm{S}$ concentrações mais elevadas foram observadas no estádio final de crescimento. Para flores de cultivares Mundo Novo e Catuaí Amarelo, Malavolta et al. (2002) encontraram quantidades médias extraídas de 69,0 kg ha-1 de Ca e 39,0 kg ha-1 de $\mathrm{Mg}$. De posse disso, Malavolta e colaboradores sugerem que a adubação do cafeeiro deve iniciar-se antes do florescimento, pois a absorção de nutrientes começa antes da antese floral. O atendimento dessa demanda de nutrientes depende da absorção pelas raízes e do transporte no xilema (Amaral, 1991; DaMatta et al., 1999; Rena, 2000), o que enfatiza a tese da antecipação da adubação, sempre considerando outros aspectos, como o uso e manejo da irrigação suplementar e a umidade no solo (Malavolta et al., 2002). 
As exigências minerais do cafeeiro variam entre variedades, de ano a ano, bem como durante o ano, sendo o período reprodutivo o de maior requerimento nutricional pela planta. $\mathrm{O}$ conhecimento da dinâmica dos nutrientes minerais nas variedades de cafeeiro, principalmente no que se refere a flores e frutos, é importante para identificar o período de maior exigência nutricional pela planta e, dessa forma, melhorar a eficiência das práticas de adubação. Além disso, conhecer as variações nos teores de nutrientes nas folhas e sua mobilização para frutos durante a fase reprodutiva de variedades de cafeeiro em diferentes ambientes é importante para auxiliar no diagnóstico do status nutricional das plantas, podendo melhorar o manejo de fertilização da cultura.

O objetivo deste trabalho foi estudar a dinâmica de Ca e Mg em frutos de cafeeiros arábicos da antese à maturação, bem como a variação desses elementos em folhas dos ramos produtivos em três níveis de adubação.

\section{MATERIAL E MÉTODOS}

O trabalho foi realizado em área experimental da Universidade Federal de Viçosa, Viçosa, MG, com altitude de $651 \mathrm{~m}$, latitude de $20^{\circ} 45^{\prime}$ 'sul e longitude de $42^{\circ} 51$ ' oeste, em talhão de café, com 4 anos de idade, implantado em Latossolo Vermelho-Amarelo distrófico, cujas características químicas são apresentadas no quadro 1 .

Foram realizados três experimentos, sendo em cada um utilizadas as variedades de Coffea arabica, Catuaí Vermelho IAC-99, Rubi MG-1192 ou Acaiá IAC-47419, plantadas em blocos ao acaso, no espaçamento de $2,0 \times 1,0 \mathrm{~m}$. Os experimentos receberam níveis de adubação e calagem designados baixo, adequado e alto, desde a implantação.

As plantas submetidas ao nível adequado de adubação receberam N, P e K com base na marcha de acúmulo, considerando-se a média de nutrientes acumulados para as cultivares Mundo Novo e Catuaí, conforme Malavolta (s.d.). Levou-se em conta ainda uma eficiência de recuperação de $90 \%$ para o N, $80 \%$ para o $\mathrm{K}$ e 70 \% para o P. O Ca e o Mg foram fornecidos via calcário dolomítico, com base em análises de solo, considerando-se $60 \%$ de saturação por bases como referência para o cafeeiro (Guimarães et al., 1999). O $\mathrm{S}$ foi fornecido como elemento acompanhante de fertilizantes nitrogenados e fosfatados. Nos níveis baixo e alto de adubação, as plantas receberam, respectivamente, 0,4 e 1,4 vezes a quantidade recomendada para o nível adequado. No ano agrícola 2001/2002 foram empregadas, em cada experimento, as doses de adubação apresentadas no quadro 2 .

Quadro 1. Características químicas do solo da área experimental nos três níveis de adubação

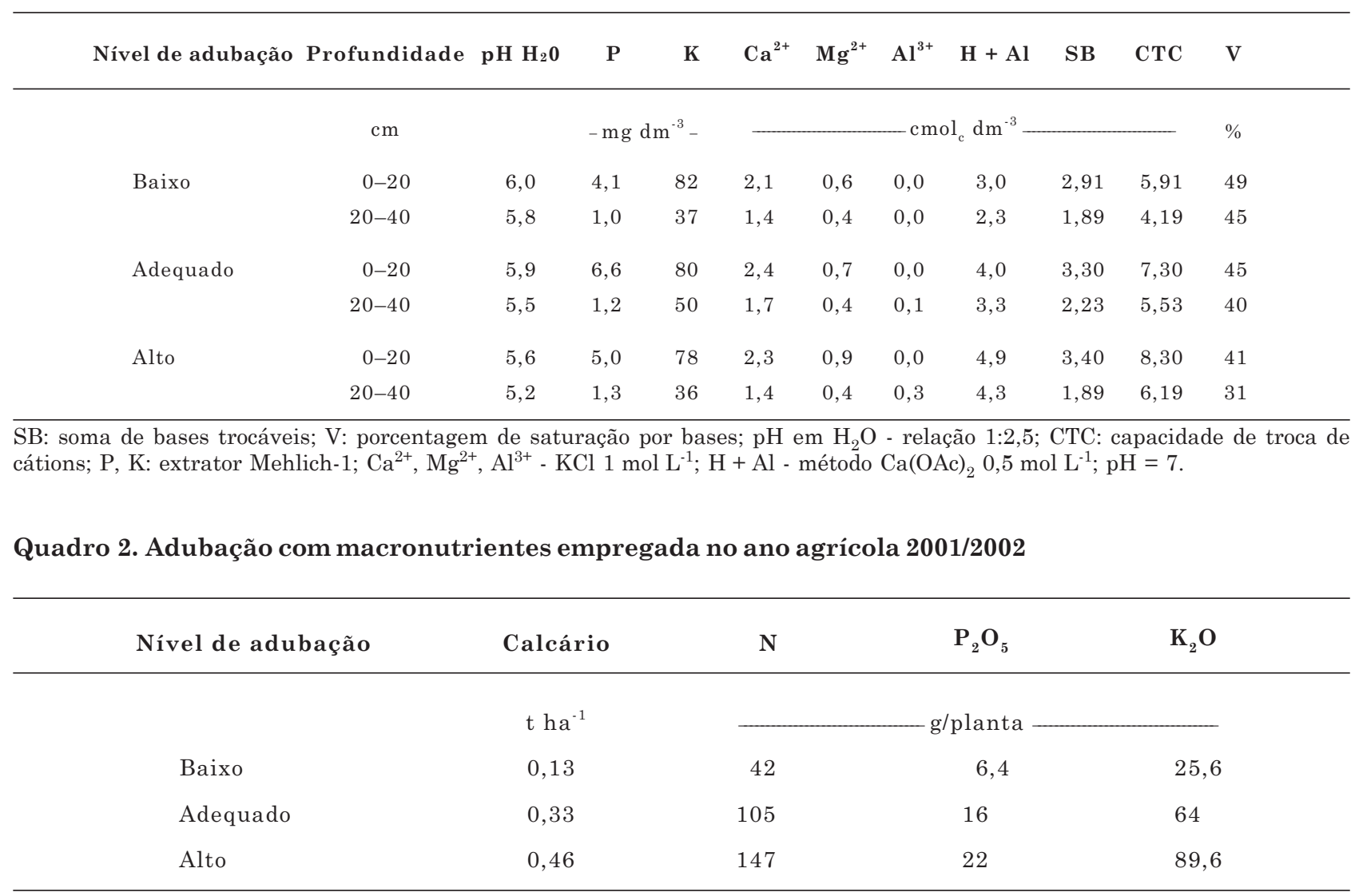


A adubação com NPK foi feita com aplicações semanais realizadas de novembro a março de forma localizada no solo, pela utilização de fertirrigação por gotejamento, com o suporte do software SISDA café (Mantovani \& Costa, 1998). Os micronutrientes Zn, $\mathrm{B}$ e $\mathrm{Cu}$ foram supridos por meio de três aplicações foliares anuais (dez., jan. e fev.), utilizando-se sulfato de zinco, ácido bórico, oxicloreto de cobre e cloreto de potássio (como adjuvante), na concentração de $4 \mathrm{~g} \mathrm{~L}^{-1}$ de cada fertilizante.

O delineamento experimental, em cada experimento (nível de adubação), foi em blocos casualizados, distribuídos em um esquema de parcelas subdivididas no tempo, sendo três variedades e 12 períodos de amostragem, com duas repetições. Cada parcela foi constituída de 25 plantas dispostas em cinco fileiras, ocupando uma área de $50 \mathrm{~m}^{2}$. Considerou-se como parcela útil o conjunto das nove plantas dispostas no centro das três fileiras centrais da parcela.

As amostragens iniciaram-se em 4 de setembro de 2001, quando houve antese floral, ocasião em que se coletaram folhas e flores, sendo este considerado como dia zero. A partir dessa data efetuaram-se coletas periódicas de folhas e frutos durante o desenvolvimento reprodutivo do cafeeiro nos seguintes períodos: aos 28 , $42,63,84,105,133,154,175,196,210$ e 224 dias após a antese. Foram coletados 100 frutos de cada parcela até a terceira amostragem, 60 frutos da quarta à setima amostragem e 20 frutos por parcela da oitava amostragem em diante. Os frutos foram colhidos aleatoriamente na parcela, de ramos pertencentes ao terço médio da planta e, as folhas, foram as correspondentes ao terceiro e quarto pares, na posição distal, de ramos com frutos, também situados no terço médio da planta. Coletaram-se durante todas as amostragens 15 folhas por parcela. A ultima amostragem foi realizada quando os frutos atingiram o ponto de maturação, ou seja, o estádio cereja, no dia 17 de abril de 2002.

O material vegetal coletado foi lavado em água deionizada e posto a secar em estufa de circulação forçada de ar a $70^{\circ} \mathrm{C}$ até atingir peso constante (Jones Junior et al., 1991), pesado, moído em moinho tipo Wiley, passado em peneira de malha de 0,841 $\mathrm{mm}$ e submetido à análise química.

Para determinação dos nutrientes Ca e Mg efetuouse a digestão nítrico-perclórica (Johnson \& Ulrich, 1959), sendo os elementos determinados por espectrofotometria de absorção atômica (AOAC, 1975).

Os dados foram submetidos a análise de variância e regressão, selecionando-se os modelos que melhor explicaram fisiologicamente a variação dos teores de $\mathrm{Ca} \mathrm{eMg}$ em folhas e frutos em função do tempo decorrido após a antese. Para explicar a variação dos elementos nos frutos, optou-se por um modelo descontínuo com duas equações de regressão.

Na seleção dos modelos, testaram-se os coeficientes das equações de regressão ajustadas com base no quadrado médio do resíduo da análise de variância, até o nível de $10 \%$ de significância. Na escolha do modelo também se considerou o coeficiente de determinação $\left(\mathrm{R}^{2}\right)$, optando-se pelo maior quando dois ou mais modelos eram significativos e explicavam o fenômeno.

\section{RESULTADOS E DISCUSSÃO}

Nas condições de Viçosa (MG), o ciclo reprodutivo do cafeeiro teve duração de 224 dias, tendo a floração ocorrido no dia 4 de setembro de 2001 e a maturação dos frutos, sido atingida no dia 17 de abril de 2002. No entanto, observou-se que o acúmulo de matéria seca não se estabilizou nesse período, apesar de os frutos apresentarem coloração de frutos maduros (Figura 1).

Durante o período de desenvolvimento, os frutos passaram por quatro estádios de desenvolvimento distintos: chumbinho, expansão rápida, crescimento suspenso e granação-maturação (Cannel, 1971a; Rena et. al., 2001), como pode ser observado na curva de acúmulo de matéria seca em frutos (Figura 1).

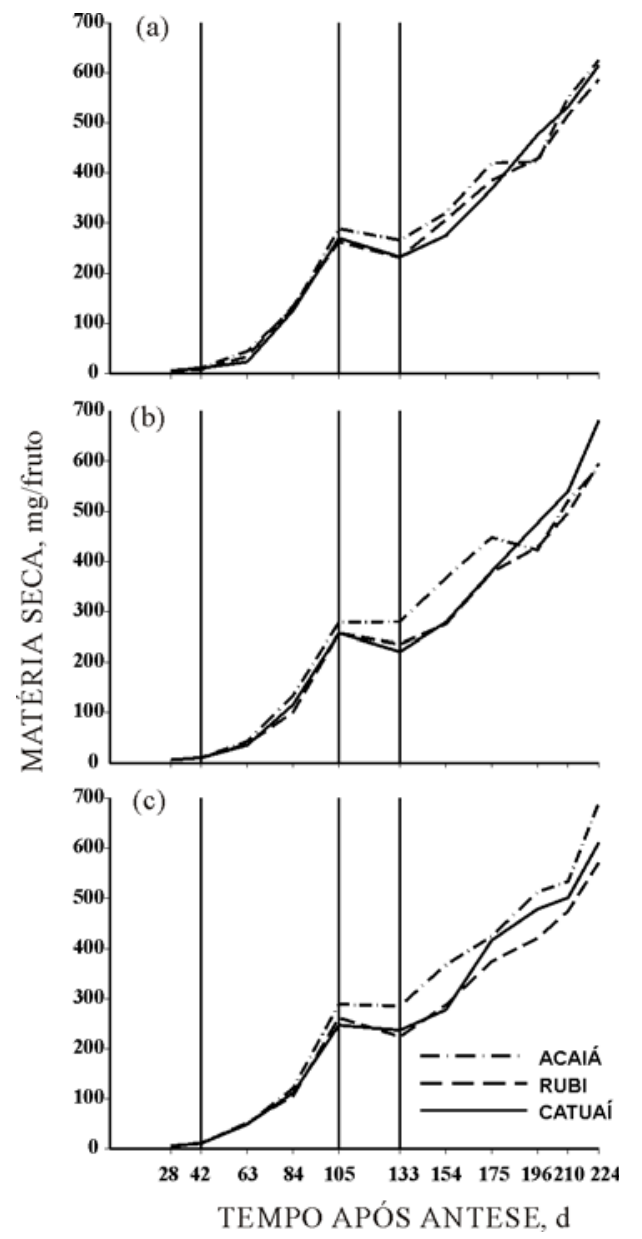

Figura 1. Acúmulo médio de matéria seca em frutos de cafeeiro durante o período reprodutivo, nos níveis baixo (a), adequado (b) e alto (c) de adubação. As linhas verticais delimitam os estádios de desenvolvimento dos frutos de chumbinho, expansão rápida, crescimento suspenso e granação-maturação. 
O primeiro estádio apresentou duração de 42 DAA (dias após antese) (Figura 1), havendo pequeno acúmulo de matéria seca, sendo este estádio denominado de chumbinho, o qual, de acordo com Rena et al. (2001), se caracteriza por intensa divisão celular e ausência de crescimento expressivo no tamanho do fruto.

O segundo estádio (Figura 1) iniciou-se com rápido aumento no conteúdo de matéria seca nos frutos, tendo duração de 42 até 105 dias após a antese (63 dias). Este estádio é conhecido como estádio de rápida expansão, no qual se observa rápido aumento do tamanho dos frutos, que ocorre devido à expansão celular. O aumento do acúmulo de matéria seca ocorre, principalmente, por deposição de substâncias de parede celular, como celulose, hemiceluloses e pectinas (Cannel, 1971a; Taiz \& Zeiger, 2004).

O estádio de crescimento suspenso teve duração de 32 dias, entre 101 e 133 DAA (Figura 1), no qual se observou acúmulo pouco expressivo de matéria seca nos frutos. É possível que este estádio seja uma fase preparatória para o estádio de granação, em que vai ocorrer a reciclagem e síntese de enzimas e compostos intermediários para serem utilizados como precursores da síntese de compostos de reservas (Taiz \& Zeiger, 2004).

Por fim, observou-se um quarto estádio de desenvolvimento dos frutos (Figura 1), dos 133 até os 224 dias após a antese, com duração de 91 dias. Neste estádio estão incluídas as últimas fases de formação dos frutos: granação e maturação, em que o acúmulo de matéria seca ocorre, principalmente, por deposição de material de reserva (Leon \& Fournier, 1962; Rena et al., 2001). Em razão de o acúmulo de matéria seca ter ocorrido até os 224 dias após a antese, não foi possível a separação dessas fases, havendo sobreposição entre elas. Esses resultados diferem dos encontrados por Chaves (1982), o qual verificou acúmulo de matéria seca no fruto de cafeeiro da variedade Catuaí até os 217 dias após o aparecimento do chumbinho, permanecendo esta quase constante até os 252 dias, momento em que se efetuou a última amostragem. No entanto, o trabalho de Chaves (1982) foi realizado em Londrina-PR, que tem características climáticas diferentes das de Viçosa e resultou em fase reprodutiva mais longa. De acordo com Camargo \& Cortez (1998), em locais de clima frio e altitudes elevadas os estádios de desenvolvimento do fruto se estendem mais, atrasando a sua maturação e colheita. A duração do ciclo do cafeeiro pode ser variável entre variedades e linhagens, de região para região, bem como na mesma região, dependendo das condições climáticas ocorridas durante a fase reprodutiva em determinado ano (Camargo et al., 2001).

As concentrações iniciais de $\mathrm{Ca}$ e de $\mathrm{Mg}$ (com exceção da variedade Rubi, no nível baixo de adubação) nas flores das variedades estudadas (Figuras 2 e 3; Quadros 4 e 5) estavam acima da faixa adequada determinada por Martinez et al. (2001): de 0,12 a
0,22 dag $\mathrm{kg}^{-1}$ de Ca e de 0,16 a 0,20 dag $\mathrm{kg}^{-1}$ de $\mathrm{Mg}$. Considerando a flor como um indicador do estado nutricional, pode-se inferir que as plantas estudadas estavam bem nutridas em Ca e Mg, mesmo no nível baixo de adubação. Malavolta et al. (2002) constataram que as flores constituem um forte dreno temporário de nutrientes, extraindo 26,2\% de Ca e 50,5\% de $\mathrm{Mg}$ (Catuaí) e 25,3 \% de Ca e 54,1 \% de Mg (Mundo Novo), em relação aos totais de $\mathrm{Ca}$ e $\mathrm{Mg}$ alocados na parte aérea da planta.

As maiores concentrações de $\mathrm{Ca}$ e $\mathrm{Mg}$ nos frutos foram encontradas no estádio de chumbinho (Figuras 2 e 3; Quadros 4 e 5). O reduzido aumento no acúmulo de matéria seca nos frutos neste estádio influenciou para que houvesse maior concentração dos elementos nos frutos. É provável que a maior concentração de Ca no estádio de chumbinho esteja relacionada à atuação deste nutriente nos processos de divisão celular e na estabilização de membranas e paredes celulares das novas células formadas
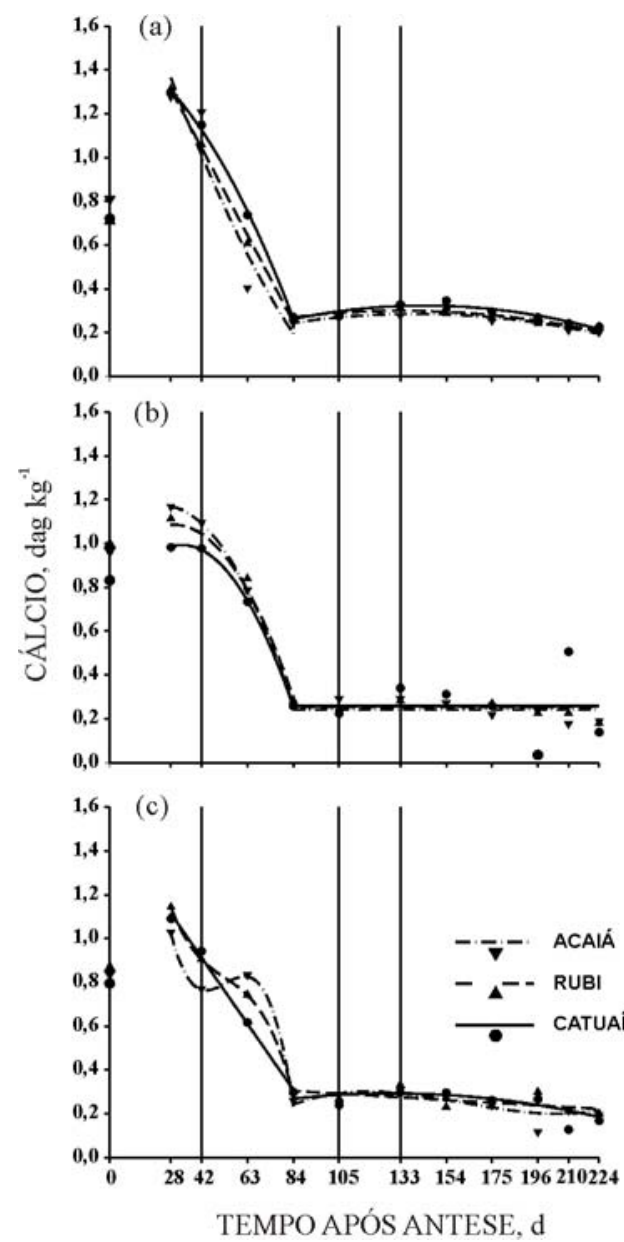

Figura 2. Concentração de cálcio em frutos de cafeeiro durante o período reprodutivo, nos níveis baixo (a), adequado (b) e alto (c) de adubação. As linhas verticais delimitam os estádios de desenvolvimento do fruto de chumbinho, expansão rápida, crescimento suspenso e granação-maturação. 

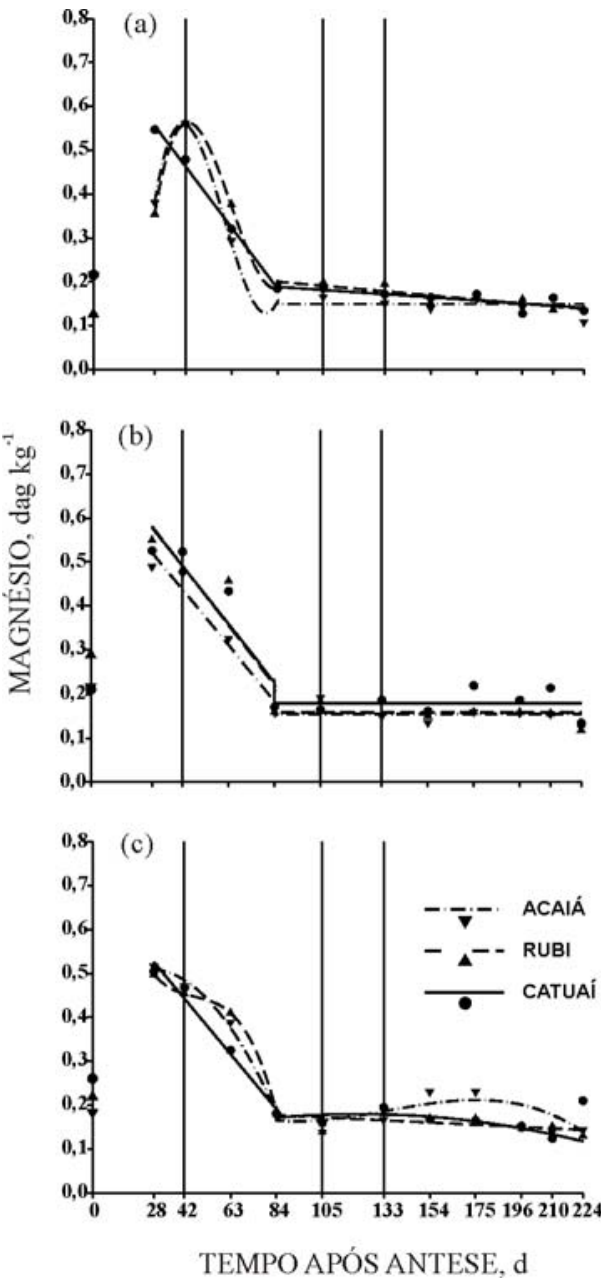

Figura 3. Concentração de magnésio em frutos de cafeeiro durante o período reprodutivo, nos níveis baixo (a), adequado (b) e alto (c) de adubação. As linhas verticais delimitam os estádios de desenvolvimento do fruto de chumbinho, expansão rápida, crescimento suspenso e granação-maturação.
(Marschner, 1995; Marenco \& Lopes, 2005). O Mg pode ter sido requerido em maior quantidade para acelerar a atividade de ATPases (Marschner, 1995), já que o fruto no estádio de chumbinho possui alta taxa respiratória (Cannell, 1971b; Rena et al., 2001).

No estádio de expansão rápida houve forte diluição do conteúdo dos nutrientes nos frutos e, conseqüentemente, redução na concentração de Ca e Mg nesses órgãos. É provável que neste estádio a translocação de Ca e Mg para os frutos venha a ocorrer por fluxo em massa, decorrente das altas taxas de translocação de água para os frutos (Ramirez et al., 2002), necessária à expansão celular (Taiz \& Zeiger, 2004; Marenco \& Lopes, 2005).

Nos estádios de crescimento suspenso e granaçãomaturação, que se estendem dos 105 aos 224 dias após a antese, observou-se pouca ou nenhuma variação nas concentrações de Ca e Mg no fruto. Verifica-se que, apesar de estar havendo acúmulo de matéria seca no fruto (Figura 1), o acúmulo de Ca e Mg nos frutos foi proporcional ao seu crescimento, permitindo pouca variação nas concentrações destes nutrientes nessas fases (Figuras 2 e 3; Quadros 4 e 5). A pequena variação nestas fases pode estar relacionada ao aumento da absorção de Ca e Mg (Souza, 1972).

No momento em que os frutos atingiram o ponto de colheita, no estádio de cereja, não se constataram diferenças acentuadas nas concentrações de $\mathrm{Ca}$ e $\mathrm{Mg}$ nos frutos das variedades estudadas. É plausível que outros fatores além da nutrição mineral, como a força de dreno causada pela produção de frutos (Quadro 3), também influenciem a composição mineral dos frutos de cafeeiro. Os teores de $\mathrm{Ca}$ e $\mathrm{Mg}$ nos frutos, em diferentes níveis de adubação, foram semelhantes aos encontrados por Ramirez et al. (2002), que constataram teores de $\mathrm{Ca}$ e $\mathrm{Mg}$ de 0,24 e 0,16 dag kg-1 em frutos maduros de cafeeiro da variedade Caturra na Costa Rica.

Pelos resultados encontrados, é possível sugerir que as práticas de adubação devem começar antes do início do estádio de expansão rápida do fruto. No caso do

Quadro 3. Produtividade de café beneficiado de três variedades de Coffea arabica submetidas a três níveis de adubação

\begin{tabular}{|c|c|c|c|}
\hline \multirow{2}{*}{ Nível de adubação } & \multicolumn{3}{|c|}{ Variedade } \\
\hline & Acaiá & Rubi & Catuaí \\
\hline \multicolumn{4}{|c|}{ Ano Agrícola 2000/2001 } \\
\hline Baixo & 12,40 & 20,70 & 19,90 \\
\hline Adequado & 18,30 & 33,20 & 30,10 \\
\hline Alto & 25,80 & 40,10 & 38,80 \\
\hline \multicolumn{4}{|c|}{ Ano Agrícola 2001/2002 } \\
\hline Baixo & 23,30 & 21,33 & 15,60 \\
\hline Adequado & 35,13 & 42,03 & 15,05 \\
\hline Alto & 44,55 & 40,48 & 39,05 \\
\hline
\end{tabular}


Quadro 4. Equações de regressão da concentração de cálcio (dag kg-1) em frutos de cafeeiro em função do tempo (d) decorrido após a antese, de acordo com o nível de adubação

\begin{tabular}{|c|c|c|c|}
\hline Variedade & Intervalo & Equação de Regressão & $\mathbf{R}^{2}$ \\
\hline \multirow{3}{*}{ Acaiá } & & Nível Baixo de Adubação & \multirow{3}{*}{0,953} \\
\hline & $28-84$ dias & $\hat{y}=2,187-0,0322^{* *} \mathrm{x}+0,000101^{0,13} \mathrm{x}^{2}$ & \\
\hline & $84-24$ dias & $\hat{y}=0,0321+0,00362^{0,18} x-0,0000126^{0,13} x^{2}$ & \\
\hline \multirow[t]{2}{*}{ Rubi } & $28-84$ dias & $\hat{y}=1,840-0,0189^{* *} \mathrm{x}$ & \multirow[b]{2}{*}{0,997} \\
\hline & 84-24 dias & $\hat{y}=0,0922+0,00310^{0,25} \mathrm{x}-0,000116^{0,18} \mathrm{x}^{2}$ & \\
\hline \multirow[t]{2}{*}{ Catuaí } & 28-84 dias & $\hat{y}=1,508-0,00341^{0,65} \mathrm{x}-0,000135^{*} \mathrm{x}^{2}$ & \multirow{2}{*}{0,998} \\
\hline & $84-24$ dias & $\hat{y}=1,234+0,0163^{*} \mathrm{x}-0,0000560^{*} \mathrm{x}^{2}$ & \\
\hline \multirow{3}{*}{ Acaiá } & & Nível Adequado de Adubação & \multirow[b]{3}{*}{0,990} \\
\hline & 28-84 dias & $\hat{y}=1,0246+0,0123^{0,59} \mathrm{x}-0,000255^{*} \mathrm{x}^{2}$ & \\
\hline & 84-24 dias & $\hat{y}=\bar{y}=0,24$ & \\
\hline \multirow[t]{2}{*}{ Rubi } & $28-84$ dias & $\hat{y}=0,855+0,0156^{0,29} \mathrm{x}-0,000246^{*} \mathrm{x}^{2}$ & \multirow{2}{*}{0,983} \\
\hline & $84-24$ dias & $\hat{y}=\bar{y}=0,25$ & \\
\hline \multirow[t]{2}{*}{ Catuaí } & $28-84$ dias & $\hat{y}=0,678+0,0190^{0,19} \mathrm{x}-0,000286^{*} \mathrm{x}^{2}$ & \multirow{2}{*}{0,872} \\
\hline & $84-24$ dias & $\hat{y}=\bar{y}=0,26$ & \\
\hline \multirow{3}{*}{ Acaiá } & & Nível Alto de Adubação & \multirow[b]{3}{*}{0,988} \\
\hline & $28-84$ dias & $\hat{y}=4,0248-0,194^{* *} \mathrm{x}+0,00377^{* *} \mathrm{x}^{2}-0,0000237^{* *} \mathrm{x}^{3}$ & \\
\hline & 84-24 dias & $\hat{y}=-0,780+0,0226^{0,16} x-0,000150^{0,17} x^{2}+0,00000031^{0,19} x^{3}$ & \\
\hline \multirow[t]{2}{*}{ Rubi } & $28-84$ dias & $\hat{y}=2,710-0,0943^{*} \mathrm{x}+0,00166^{*} \mathrm{x}^{2}-0,0000105^{*} \mathrm{x}^{3}$ & \multirow{2}{*}{0,991} \\
\hline & 84-24 dias & $\hat{y}=0,353-0,000586^{\circ} \mathrm{x}$ & \\
\hline \multirow[t]{2}{*}{ Catuaí } & 28-84 dias & $\hat{y}=1,516-0,0143^{* *} \mathrm{x}$ & \multirow[b]{2}{*}{0,987} \\
\hline & $84-24$ dias & $\hat{y}=0,0915+0,00311^{0,24} \mathrm{x}-0,0000120^{0,16} \mathrm{x}^{2}$ & \\
\hline
\end{tabular}

$* *{ }^{*} \mathrm{e}^{\circ}$, significativos a 1,5 e $10 \%$, respectivamente.

Quadro 5. Equações de regressão da concentração de magnésio (dag kg-1) em frutos de cafeeiro em função do tempo decorrido após a antese, de acordo com o nível de adubação

\begin{tabular}{|c|c|c|c|}
\hline Variedade & Intervalo & Equação de Regressão & $\mathbf{R}^{2}$ \\
\hline \multirow{3}{*}{ Acaiá } & & Nível Baixo de Adubação & \multirow[b]{3}{*}{0,979} \\
\hline & $28-84$ dias & $\hat{y}=-2,000+0,151^{* *} \mathrm{x}-0,00281^{* *} \mathrm{x}^{2}+0,0000157^{* *} \mathrm{x}^{3}$ & \\
\hline & $84-24$ dias & $\hat{y}=\bar{y}=0,15$ & \\
\hline \multirow[t]{2}{*}{ Rubi } & $28-84$ dias & $\hat{y}=-1,760+0,130^{* *} \mathrm{x}-0,00229^{* *} \mathrm{x}^{2}+0,0000121^{* *} \mathrm{x}^{3}$ & \multirow{2}{*}{0,995} \\
\hline & $84-24$ dias & $\hat{y}=0,237-0,000444^{\circ} \mathrm{x}$ & \\
\hline \multirow[t]{2}{*}{ Catuaí } & $28-84$ dias & $\hat{y}=0,741-0,00661^{* *} \mathrm{x}$ & \multirow{2}{*}{0,993} \\
\hline & $84-24$ dias & $\hat{y}=0,217-0,000341^{0,18} \mathrm{x}$ & \\
\hline \multirow[t]{2}{*}{ Acaiá } & $28-84$ dias & $\begin{array}{l}\text { Nível Adequado de Adubação } \\
\hat{y}=0,690-0,00602^{0,11} \mathbf{x}\end{array}$ & \multirow[b]{2}{*}{0,969} \\
\hline & $84-24$ dias & $\hat{y}=\bar{y}=0,15$ & \\
\hline \multirow[t]{2}{*}{ Rubi } & $28-84$ dias & $\hat{y}=0,461-0,00644^{\circ} \mathrm{x}$ & \multirow[b]{2}{*}{$0,92 \mathrm{~s}$} \\
\hline & $84-24$ dias & $\hat{y}=\bar{y}=0,15$ & \\
\hline \multirow[t]{2}{*}{ Catuaí } & $28-84$ dias & $\hat{y}=0,754-0,00628^{\circ} \mathrm{x}$ & \multirow[b]{2}{*}{0,920} \\
\hline & $84-24$ dias & $\hat{y}=\bar{y}=0,18$ & \\
\hline \multirow[t]{2}{*}{ Acaiá } & $28-84$ dias & $\begin{array}{c}\text { Nível Alto de Adubação } \\
\hat{v}=0,444+0,00502^{0,13} \mathrm{X}-0,0000970^{* *} \mathrm{x}^{2}\end{array}$ & \multirow[b]{2}{*}{0,966} \\
\hline & 84-24 dias & $\hat{y}=0,506-0,00889^{0,21} \mathrm{x}+0,0000727^{0,13} \mathrm{x}^{2}-0,00000018^{\circ} \mathrm{x}^{3}$ & \\
\hline \multirow[t]{2}{*}{ Rubi } & $28-84$ dias & $\hat{y}=0,941-0,0292^{0,13} \mathrm{x}+0,000597^{\circ} \mathrm{x}^{2}-0,00000424^{*} \mathrm{x}^{3}$ & \multirow{2}{*}{0,990} \\
\hline & $84-24$ dias & $\hat{y}=0,197-0,000241^{\circ} \mathrm{x}$ & \\
\hline \multirow[t]{2}{*}{ Catuaí } & $28-84$ dias & $\hat{y}=0,694-0,00599 * * x$ & \multirow[b]{2}{*}{0,938} \\
\hline & $84-24$ dias & $\hat{v}=0,0911+0,00145^{0,22} \mathrm{x}-0,00000597^{0,11} \mathrm{x}^{2}$ & \\
\hline
\end{tabular}

$* *{ }^{*} \mathrm{e}^{\mathrm{o}}$, significativos a 1,5 e $10 \%$, respectivamente. 
ano agrícola 2001/02, em Viçosa, o fornecimento de nutrientes deveria ter sido iniciado antes dos $42 \mathrm{DAA}$ (16/10/2001). Malavolta et al. (2002) também enfatizam o fato de que as adubações do cafeeiro devemse iniciar no princípio do estádio reprodutivo. No entanto, devem-se considerar também outros aspectos, como o uso e manejo de irrigação suplementar e a umidade no solo, para efetuar as adubações.

O padrão das curvas de variação de Ca e Mg nas folhas do $3^{\circ}$ e $4^{\circ}$ pares dos ramos produtivos (Figuras 4 e 5; Quadros 6 e 7) diferenciou-se do constatado por Souza (1972), que foi o aumento contínuo da concentração dos elementos nas folhas do $3^{\circ}$ e $4^{\circ}$ pares de ramos produtivos da variedade Mundo Novo. Confrontado com o observado por Chaves (1982), verifica-se que o padrão da curva de variação de Ca nos frutos também se diferencia, porém, para $\mathrm{Mg}$, os resultados se assemelham.
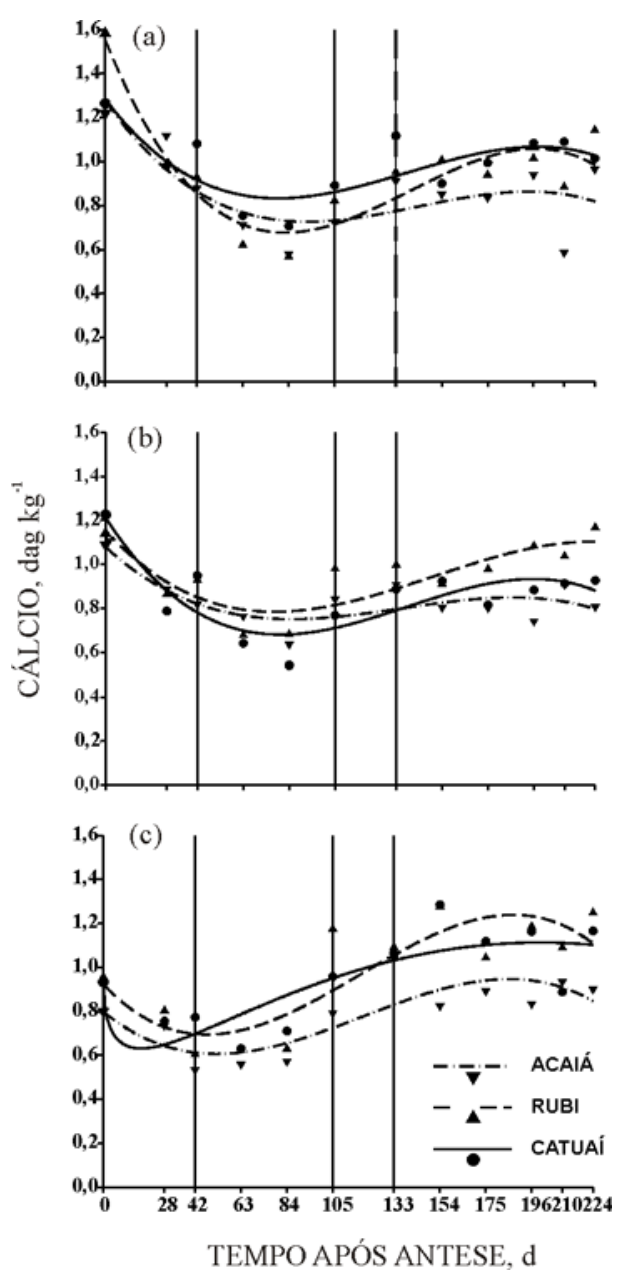

Figura 4. Concentração de cálcio em folhas de cafeeiro durante o período reprodutivo, nos níveis baixo (a), adequado (b) e alto (c) de adubação. As linhas verticais delimitam os estádios de desenvolvimento do fruto de chumbinho, expansão rápida, crescimento suspenso e granação-maturação.
No momento da floração, as folhas das variedades estudadas mostraram tendência de apresentar menores concentrações de $\mathrm{Ca}$ e $\mathrm{Mg}$ no nível alto de adubação (Figuras 4 e 5; Quadros 6 e 7). Isso pode estar relacionado à floração mais intensa nesse nível de adubação, que resultou em diluição do nutriente. Além disso, como o calcário possui baixa solubilidade e reação lenta no solo (Alvarez V. \& Ribeiro, 1999), pode não ter havido disponibilização suficiente do Ca e Mg aplicados via calcário, o que foi acentuado pela baixa concentração dos nutrientes no solo (Quadro 1). Outra justificativa estaria no fato de a produção de frutos da safra anterior ter demandado mais $\mathrm{Ca}$ e $\mathrm{Mg}$, deixando as plantas do nível alto de adubação com menores teores foliares dos elementos (Quadro 3).

$\mathrm{Na}$ fase reprodutiva, após atingirem a mínima concentração, as concentrações de Ca e Mg nas folhas aumentaram novamente até que se alcançasse uma
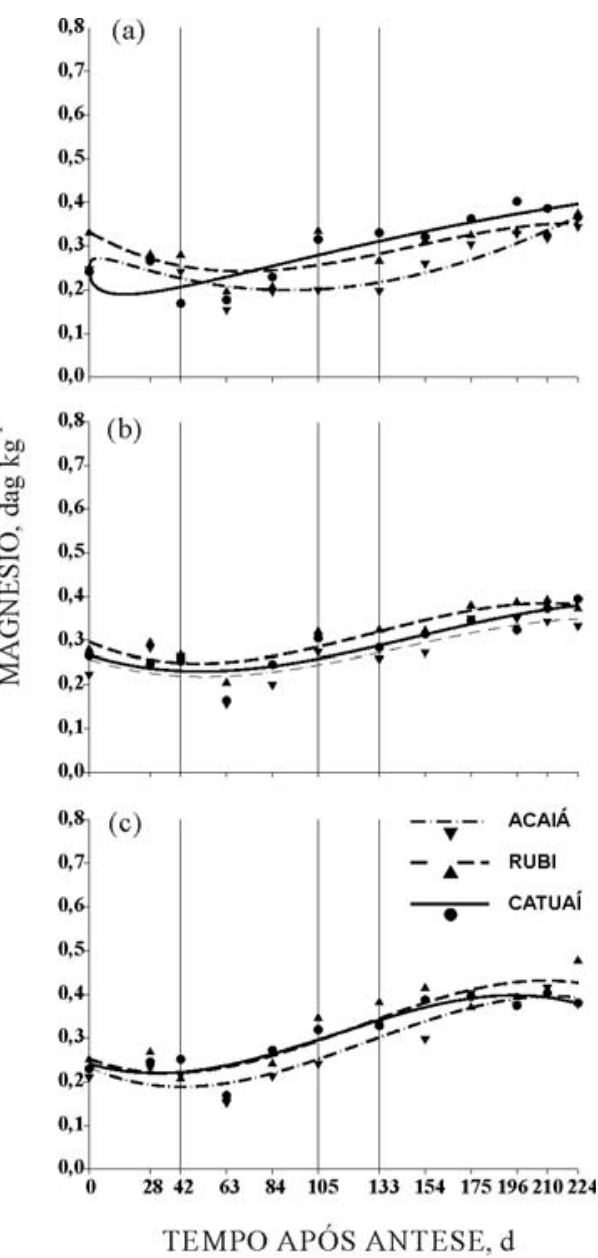

Figura 5. Concentração de magnésio em folhas de cafeeiro durante o período reprodutivo, nos níveis baixo (a), adequado (b) e alto (c) de adubação. As linhas verticais delimitam os estádios de desenvolvimento do fruto de chumbinho, expansão rápida, crescimento suspenso e granação-maturação. 
Quadro 6. Equações de regressão da concentração de cálcio (dag kg-1) em folhas de cafeeiro em função do tempo (d) decorrido após a antese, de acordo com o nível de adubação

\begin{tabular}{|c|c|c|}
\hline Variedade & Equação de Regressão & $\mathbf{R}^{2}$ \\
\hline \multicolumn{3}{|c|}{ Nível Baixo de Adubação } \\
\hline Acaiá & $\hat{y}=1,279-0,0141^{*} \mathrm{x}+0,000113^{*} \mathrm{x}^{2}-0,000000264^{\circ} \mathrm{x}^{3}$ & 0,583 \\
\hline Rubi & $\hat{y}=1,555-0,0247 * * \mathrm{x}+0,000212^{* *} \mathrm{x}^{2}-0,000000507 * * \mathrm{x}^{3}$ & 0,838 \\
\hline Catuaí & $\hat{y}=1,285-0,0131^{* *} \mathrm{x}+0,000116^{*} \mathrm{x}^{2}-0,000000280^{*} \mathrm{x}^{3}$ & 0,631 \\
\hline \multicolumn{3}{|c|}{ Nível Adequado de Adubação } \\
\hline Rubi & $\hat{y}=0,121-0,0102 * x+0,0000923^{*} x^{2}-0,000000209^{*} x^{3}$ & 0,689 \\
\hline Catuaí & $\hat{y}=1,212-0,0153^{* *} \mathrm{x}+0,000135 * * \mathrm{x}^{2}-0,000000326^{*} \mathrm{x}^{3}$ & 0,715 \\
\hline \multicolumn{3}{|c|}{ Nível Alto de Adubação } \\
\hline Acaiá & $\hat{y}=0,799-0,00820 * \mathrm{x}+0,000101 * \mathrm{x}^{2}-0,000000284^{*} \mathrm{x}^{3}$ & 0,655 \\
\hline Rubi & $\hat{y}=0,923-0,0105^{*} \mathrm{x}+0,000139^{*} \mathrm{x}^{2}-0,000000395^{*} \mathrm{x}^{3}$ & 0,702 \\
\hline Catuaí & $\hat{y}=0,951-0,170^{*} \mathrm{x}^{0,5}+0,0265^{*} \mathrm{x}-0,000968^{\circ} \mathrm{x}^{1,5}$ & 0,643 \\
\hline
\end{tabular}

**, * $\mathrm{e}^{\circ}$, significativos a 1,5 e $10 \%$, respectivamente.

Quadro 7. Equações de regressão da concentração de magnésio ( $\left.\mathrm{dag} \mathrm{kg}^{-1}\right)$ em folhas de cafeeiro em função do tempo (d) decorrido após a antese, de acordo com o nível de adubação

\begin{tabular}{|c|c|c|}
\hline Variedade & Equação de Regressão & $\mathbf{R}^{2}$ \\
\hline \multicolumn{3}{|c|}{ Nível Baixo de Adubação } \\
\hline Acaiá & $\hat{y}=0,251+0,0211^{0,13} \mathbf{x}^{0,5}-0,00612^{*} \mathrm{x}+0,000349^{*} \mathrm{x}^{1,5}$ & 0,815 \\
\hline Rubi & $\hat{y}=0,332-0,00286^{*} \mathrm{x}+0,0000267^{*} \mathrm{x}^{2}-0,0000000605^{*} \mathrm{x}^{3}$ & 0,632 \\
\hline Catuaí & $\hat{y}=0,248-0,0312^{\circ} \mathbf{x}^{0,5}+0,00463^{0,13} \mathbf{x}-0,000125^{0,24} \mathbf{x}^{1,5}$ & 0,804 \\
\hline \multicolumn{3}{|c|}{ Nível Adequado de Adubação } \\
\hline Acaiá & $\hat{y}=0,257-0,00161^{0,15} x+0,0000187^{0,13} x^{2}-0,0000000435^{0,18} x^{3}$ & 0,830 \\
\hline Rubi & $\hat{y}=0,297-0,00212^{*} \mathrm{x}+0,0000262^{*} \mathrm{x}^{2}-0,0000000672^{0,14} \mathrm{x}^{3}$ & 0,832 \\
\hline Catuaí & $\hat{y}=0,267-0,00154^{0,11} \mathrm{x}+0,0000182^{*} \mathrm{x}^{2}-0,0000000^{0,14} \mathrm{x}^{3}$ & 0,798 \\
\hline \multicolumn{3}{|c|}{ Nível Alto de Adubação } \\
\hline Acaiá & $\hat{y}=0,233-0,00226 * x+0,0000319 * x^{2}-0,0000000 * x^{3}$ & 0,889 \\
\hline Rubi & $\hat{y}=0,252-0,00188^{0,15} x+0,0000303^{\circ} x^{2}-0,0000000^{\circ} x^{3}$ & 0,811 \\
\hline Catuaí & $\hat{y}=0,241-0,00147^{0,11} \mathrm{x}+0,0000275^{*} \mathrm{x}^{2}-0,0000000813^{*} \mathrm{x}^{3}$ & 0,878 \\
\hline
\end{tabular}

**, ${ }^{*} \mathrm{e}^{\circ}$, significativos a 1,5 e $10 \%$, respectivamente.

máxima concentração de cada elemento (Figuras 4 e 5; Quadros 6 e 7). Essa máxima concentração ocorreu no estádio de granação-maturação do fruto, quando supõe-se ter havido dreno de Ca e Mg para os órgãos reprodutivos. Assim, o aumento da absorção de Ca e
Mg pelas raízes, nessa época, não teria sido impulsionado somente pela intensidade de dreno dos frutos, mas também por outros fatores fisiológicos que regulam também a partição dos nutrientes para as folhas. 
Aos 224 dias, as concentrações foliares de Ca em todas as variedades e de Mg nas variedades Acaiá e Rubi no nível alto de adubação apresentaram os maiores valores (Figuras 4 e 5; Quadros 6 e 7). Com o avanço do período reprodutivo, as reações do calcário no solo foram se tornando mais efetivas, disponibilizando maior quantidade de Ca e Mg para absorção e transporte para as folhas, e os frutos foram deixando de ser os drenos preferenciais para estes nutrientes.

Ao final do período reprodutivo verificou-se que somente as concentrações de Ca nas folhas no nível alto de adubação foram superiores aos valores observados no início do período reprodutivo (Figura 4; Quadro 6). Nos níveis baixo e adequado de adubação, as concentrações iniciais de Ca foram as maiores observadas ao longo do período reprodutivo, superando até mesmo as finais. Isso indica que nestes níveis o fornecimento de $\mathrm{Ca}$ pode ter sido inadequado às exigências nutricionais das variedades. Quanto ao $\mathrm{Mg}$ (Figura 5; Quadro 7), observou-se que, independentemente do nível de adubação, as concentrações finais de $\mathrm{Mg}$ nas três variedades foram superiores às iniciais. Assim, pode-se inferir que o fornecimento de $\mathrm{Mg}$ foi adequado no suprimento nutricional das variedades em todos os níveis de adubação.

Por ser a análise foliar importante ferramenta para o diagnóstico nutricional das plantas, em cafeeiro, ela deve ser realizada antes da fase de rápida expansão do fruto (Martinez et al., 1999). Nas condições de Viçosa, no ano agrícola 2001/2002, a análise foliar deveria ter sido realizada antes de 16 de outubro, momento em que se iniciou a fase de expansão rápida e o acúmulo de nutrientes em frutos. Floradas mais tardias podem retardar o início da fase de expansão do fruto (Camargo et al., 2001), razão pela qual estudos adicionais são necessários para o estabelecimento de épocas adequadas para análise foliar, considerando as fases fenológicas dos frutos para diferentes variedades e condições climáticas.

\section{CONCLUSÕES}

1. Os níveis de adubação influenciaram a concentração de $\mathrm{Ca}$ e $\mathrm{Mg}$ em frutos e folhas das variedades de Coffea arabica ao longo do período reprodutivo. Contudo, as concentrações de $\mathrm{Ca}$ e $\mathrm{Mg}$ em folhas e frutos não foram influenciadas somente pelos níveis de adubação empregados, mas também por outros fatores que determinam a taxa de distribuição dos elementos minerais nas plantas de cafeeiros, como a carga pendente de frutos.

2. O padrão das curvas de variação de $\mathrm{Ca}$ e $\mathrm{Mg}$ durante os estádios de formação dos frutos foi semelhante entre as variedades, bem como nos níveis de adubação.

\section{LITERATURA CITADA}

ALVAREZ V., V.H. \& RIBEIRO, A.C. Calagem. In: RIBEIRO, A.C.; GUIMARÃES, P.T.G.; ALVAREZ V., V.H., ed. Recomendações para o uso de corretivos e fertilizantes em Minas Gerais, $5^{\text {a }}$ Aproximação. Viçosa,MG, Comissão de Fertilidade do Solo do Estado de Minas Gerais CFSEMG. 1999. p.44-60.

AMARAL, J.A.T. Crescimento vegetativo estacional do cafeeiro e suas interações com fontes de nitrogênio, fotoperíodo, fotossíntese e assimilação do nitrogênio. Viçosa, MG, Universidade Federal de Viçosa, 1991. 139p. (Tese de Doutorado)

AOAC. Official methods of analysis. 12.ed. Washington, D.C., 1975. $1094 \mathrm{p}$.

CAMARGO, A.P. As oito fases fenológicas da frutificação do cafeeiro. In: CONGRESSO BRASILEIRO DE PESQUISAS CAFEEIRAS, 24., Rio de Janeiro, 1998. Trabalhos apresentados. Rio de Janeiro, PROCAFÉ, 1998. v.1. p.41-42

CAMARGO, A.P. \& CAMARGO, M.B.P. Definição e esquematização das fases fenológicas do cafeeiro arábica nas condições tropicais do Brasil. Bragantia, 60:65-68, 2001.

CAMARGO, M.B.P.; PEDRO JÚNIOR, M.J.; CAMARGO, A.P.; FAHL, J.I.; FAZUOLI, L.C. \& SANTOS, M.A. Modelo agrometeorológico de estimativa da época da plena floração do cafeeiro arábica em condições tropicais. In: SIMPÓSIO BRASILEIRO DE PESQUISA DOS CAFÉS DO BRASIL, 2., Brasília, 2001. Anais. Brasília, Embrapa Café, 2001. CD-ROM

CANNEL, M.G.R. Changes in the respiration and growth rates of developing fruits of Coffea arabica L.J. Hortic. Sci., 46:263-272, 1971b.

CANNEL, M.G.R. Seasonal patterns of growth and development of arabica coffee in Kenya. PartIV. Effects of seasonal differences in rainfall on bean size. Kenya Coffee, 36:175-180, 1971a.

CHAVES, J.C.D. Concentração de nutrientes em frutos e folhas e exportação de nutrientes pela colheita durante um ciclo produtivo do cafeeiro (Coffea arabica L. CV. Catuaí). Piracicaba, Escola Superior de Agricultura Luiz de Queiroz, 1982. 131p. (Tese de Mestrado)

CONAB. Safra - 2006/2007 final. Disponível em: < http:// www.conab.gov.br/conabweb/download/safra/ 3levantamentocafesafra200607.pdf>. Acesso em 14 de setembro de 2006 .

DA MATTA, F.M.; AMARAL, J.A.T.\& RENA, A.B. Growth periodicity in trees of Coffea arabica L. in relation to nitrogen supply and nitrate reductase activity. Field Crops Res., 60:223-229, 1999.

FAZUOLI, L.C.; MEDINA FILHO, H.P.; GONÇALVES, W.; GUERREIRO FILHO, O. \& SILVAROLLA, M.B. Melhoramento do cafeeiro: variedades tipo arábicas obtidas no Instituto Agronômico de Campinas. In: ZAMBOLIM, L. O estado da arte de tecnologias na produção de café. Viçosa, MG, Universidade Federal de Viçosa, 2002. p.163-215. 
GOUVEIA, N.M. Estudo da diferenciação e crescimento das gemas florais de Coffea arabica L.: observação sobre antese e maturação dos frutos. Campinas, Universidade de Campinas, 1984. 237p. (Tese de Mestrado)

GUIMARÃES, P.T.G.; GARCIA, A.W.R.; ALVAREZ V., V.H.; PREZOTTI, L.C.; VIANA, A.S.; MIGUEL, A.E.; MALAVOLTA, E.; CORRÊA, J.B., LOPES, A.S.; NOGUEIRA, F.D.\& MONTEIRO, A.V.C. Cafeeiro. In: RIBEIRO, A.C.; GUIMARÃES, P.T.G.; ALVAREZ V., V.H., ed. Recomendações para o uso de corretivos e fertilizantes em Minas Gerais, $5^{\text {a }}$ Aproximação. Viçosa, MG, Comissão de Fertilidade do Solo do Estado de Minas Gerais CFSEMG. 1999. p.289-302.

JOHNSON, C.M. \& ULRICH, A. Analytical methods for use in plants analyses. Los Angeles, University of California, 1959. v.766. p.32-33.

JONES JUNIOR, J.B.; WOLF, B. \& MILLS, H.A. Plant analysis handbook: a practical sampling, preparation, analysis, and interpretation guide. Athens, Micro-Macro Publishing, 1991. 213p.

LEON, J. \& FOURNIER, L. Crecimento y desarollo del fruto de Coffea arabica. Turrialba, 12:65-74, 1962.

MALAVOLTA, E. Nutrição, adubação e calagem do cafeeiro. São Paulo, Copas Fertilizantes, s.d. 43p.

MALAVOLTA, E.; FAVARIN, J.L.; MALAVOLTA, M.; CABRAL, C.P.; HEINRICHS, R.\& SILVEIRA, J.S.M. Repartição de nutrientes nos ramos, folhas e flores do cafeeiro. R. Pesq. Agropec. Bras., 37:1017-1022, 2002.

MANTOVANI, E.C. \& COSTA, L.C. Manual do SISDA 2.0 Workshop Internacional sobre Manejo Integrado das Culturas e Recursos Hídricos. Viçosa, MG, 1998. 153p.

MARENCO, R.A.\& LOPES, N.F. Fisiologia vegetal: fotossíntese, respiração, relações hídricas e nutrição mineral. Viçosa, MG, Universidade Federal de Viçosa, 2005. $451 \mathrm{p}$.

MARSCHNER, H. Mineral nutrition of higher plants. 2.ed. New York, Academic Press, 1995. 889p.
MARTINEZ, H.E.P.; CARVALHO, J.G. \& SOUZA, R.B. Diagnose foliar. In: RIBEIRO, A.C.; GUIMARÃES, P.T.G.\& ALVAREZ V., V.H., eds. Recomendações para o uso de corretivos e fertilizantes em Minas Gerais. $5^{\text {a }}$ Aproximação. Viçosa, MG, Comissão de Fertilidade do Solo do Estado de Minas Gerais - CFSEMG, 1999. p.144168.

MARTINEZ, H.E.P.; SOUZA, R.B.; ABADIA, J.B. ALVAREZ V., V.H. \& SANZ, M. Coffee-tree analysis as a mean of nutritional diagnosis. J. Plant Nutr., 26:1467-1482, 2003.

MATIELLO, J.B.; SANTINATO, R.; GARCIA, A.W.R.; ALMEIDA, S.R. \& FERNANDES, D.R. Cultura de café no Brasil: novo manual de recomendações. Rio de Janeiro, MAPA/PROCAFE, 2005. 438p.

MORAES, F.R.P. \& CATANI, R.A. A absorção de elementos minerais pelo fruto do cafeeiro durante o seu desenvolvimento. In: REUNIÃO ANUAL DA SBPC. RESUMOS DAS COMUNICAÇÕES À XVI REUNIÃO ANUAL DA SBPC. Ci.Cult.,16:142, 1964.

RAMÍREZ, F.; BERTSCH, F. \& MORA, L. Consumo de nutrimentos por los frutos y bandolas de cafe Caturra durante um ciclo de desarrollo y maduracion en Aquiares, Turrialba, Costa Rica. Agron. Costarrcence, 26:33-42, 2002.

RENA, A.B. Adubação de inverno do cafeeiro. Lavras, Epamig/ CBP\&D-Café, 2000. 2p. (Circular Técnica, 120)

RENA, A.B.; BARROS, R.S. \& MAESTRI, M. Desenvolvimento reprodutivo do cafeeiro. In: ZAMBOLIM L. Tecnologias de produção de café com qualidade. Viçosa, MG, Universidade Federal de Viçosa, 2001. p.101-128.

SOUZA, V.H.S. Variações no teor de alguns elementos minerais nas folhas e frutos de café (Coffea arabica L. Var. Mundo Novo).Viçosa, MG, Universidade Federal de Viçosa, 1972. 43p. (Tese de Mestrado)

TAIZ, L. \& ZEIGER, E. Fisiologia vegetal. 3.ed. Porto Alegre, Artmed, 2004. 719p. 